\title{
Article
}

\section{Passaggio peak coefficient as an indicator of singer advancement}

\author{
Mateusz Gawlik ${ }^{1,+, \ddagger(\mathbb{D}, \text { Wiesław Wszołek }}{ }^{1, \ddagger}$ \\ 1 AGH University of Science and Technology \\ * Correspondence: mateogawel@gmail.com
}

\begin{abstract}
In the last few years, researchers have paid increasing attention to singing voice evaluations.In their studies, they observed changes in the vibrations of the vocal folds during the transi-tion of registers. Additionally, they also found that these changes are less visible and audiblein the case of skilled singers. In order to confirm this theory we defined a new parameter,the Passaggio Peak Coefficient (PPC), obtained from an EGG signal to analyse pitch andopen quotient jump characteristics during the transition of vocal registers among 21 femaleand male choir members with different singing skills. The Kruskal-Wallis test proved thatit is possible to distinguish vocal skills, based on the ability to smoothen transitions amongfemale singers at a 5\% significance level.
\end{abstract}

Keywords: passaggio, pitch, glissando, singing voice

\section{Introduction}

High-quality singing is not a straightforward task. It takes a couple of years of training to achieve the experience and skills to be classified as a good singer. Moreover, there are many ways to assess one's singing voice. In order to classify vocal skills pedagogues use such terms as intonation, timbre, enunciation, tessitura etc. One of the features that distinguish a high and low quality voice is the vocal registers transition way.

There are several definitions of vocal registers. Additionally, experts do not agree upon number of vocal registers. Each register utilizes a particular part of the vocal folds to produce sound. The most common registers theory is defined as chest, mixed and head voice. Vocal registers are ofter defined as a set of sounds produced by one mechanism [1]. Another definitions is a series of homogeneous in pitch and loudness vocal tones [2].

The crucial term related with vocal registers and singing skills is passaggio. The passaggio is a Italian term used in classical singing to describe the vocal register transition area between. During register transition changes in the length and thickness of vocal folds occur. It is related to the moment of registers change. Classical italian school defines primo, secondo and zona di passaggio in the male voice, and primo and secondo passaggio in the female voice. The pivotal notes between passaggio depends on voice type. It normally occurs between $\mathrm{D}$ and $\mathrm{G}$ sounds. Table 1 shows passaggio thresholds used for this study. One of the goals of classical voice training is to sing throughout the passagio with an even timbre.

Table 1. Passagio thresholds for alto and soprano voices.

\begin{tabular}{ccc}
\hline & Alto & Soprano \\
\hline 1st passaggio & E4 & G4-A4 \\
2nd passaggio & D5 & D5 \\
\hline
\end{tabular}

The term laryngeal vibratory mechanisms was introduced by [3] as the glottis configurations that enable full vocal range generation [1]. There are four laryngeal vibratory 

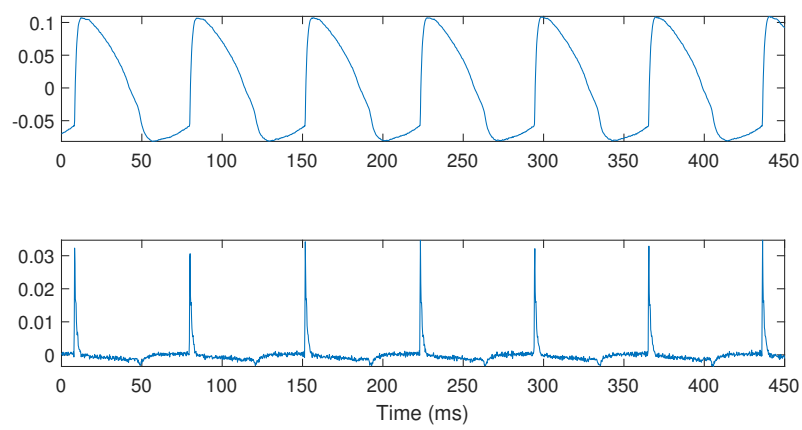

Figure 1. Example of laryngeal vibratory mechanism M1. Upper plot shows EGG signal, the bottom one its derivative.
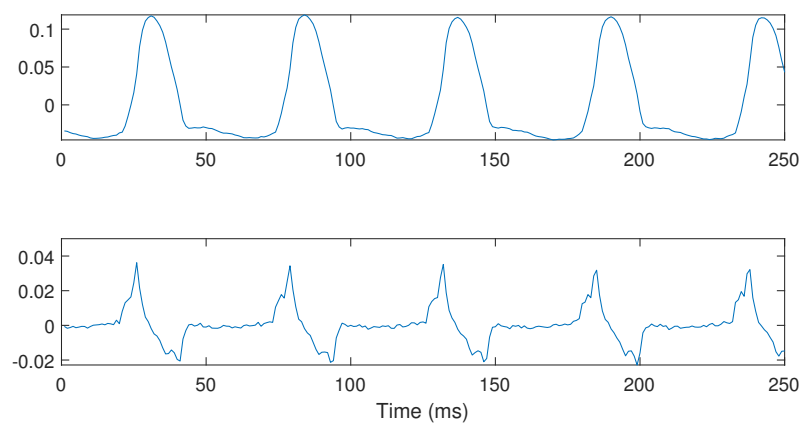

Figure 2. Example of laryngeal vibratory mechanism M2. Upper plot shows EGG signal, the bottom one its derivative.

mechanisms: M0-M3 whose ranges often overlap each other. [4] describes laryngeal vibratory patterns. According to this we used following definitions to distinguish laryngeal vibratory mechanisms in this work:

- M0 has a short opening phase, irregular oscillation, short, thick and loose vocal folds. The lowest notes are produced in that mechanism.

- M1 is characterized by thick vocal folds, vibrating in their entire length. This oscilations are phase shifted.

- $\quad$ M2 is characterized by a lower vocal folds contact area, associated only with vibrations of the vocal folds upper parts. There is no phase shift of those vibrations.

- $\quad$ M3 is used to generate the highest tones. The vocal folds are thin, very tense and the amplitude of their vibrations is lower than in M2.

Table 2. Singers group recorded for this study description,

\begin{tabular}{ccc}
\hline M0 & M1 & M2 \\
M3 & & \\
\hline $\begin{array}{c}\text { Vocal fry } \\
\text { whistle }\end{array}$ & Chest and male head & Falsetto and female head \\
\hline
\end{tabular}

Figures 1 and 2 present examples of electroglottographic (egg) and its derivative (degg) signals produced in M1 and M2 laryngeal vibratory mechanisms. There are several significant differences between these two vibrations way present [5-7]. At first, egg signal for M1 is more asymmetric, than M2. Secondly, open phase in M1 is noticeable longer. Finally, the negative peaks of degg is comparable with the positive peak (vocal folds gaped time) only in M2. Vocal folds vibratory patterns and its influence on vocal registers were introduced in [6] and collected in table ??.

Skilled singers can move through vocal ranges and dynamics smoothly. It causes no vocal breaks during singing. One of the main tasks of vocal training is to eliminate that 
vocal breaks during register transitions. It causes structural changes in the glottis and the vibratory characteristics of vocal folds inaudible [4-6]. [8] describes singers' strategies to achieve this aim.

As mentioned above skilled singers can smooth transitions to make the structural changes of vocal folds vibrations way inaudible. This is the vocal education crucial part. Incorrect vocal technique leads to audible differences in the timbre between different registers. It is possible to detect this differences by a pitch jumps, observed in $[9,10]$. Sudden, instantaneous pitch peaks were also noticed in [11,12]. Additionally, [13,14] noted differences in egg and degg amplitudes and single period characterizations during passaggio.

As presented above, there are couple of studies related with register transition and singing differences between skilled and unskilled singer strategies during passaggio. In this study we tested the hypothesis, that it is possible to assess quality of singing voice by pitch jumps parametrization during passaggio. However there is no measurable parameter to describe it. According to this we defined a newly parameter - Passaggio Peak Coefficient (PPC). Additionally, we tested its usefulness in a singing technique assessments.

\section{Materials and Methods}

Recording session

Table 3. Singers group recorded for this study description,

\begin{tabular}{ccc}
\hline No. & Voice type & Symbol \\
\hline p1 & Alto & proper \\
p4 & Soprano & inproper \\
p6 & Soprano & inproper \\
p10 & Soprano & inproper \\
p13 & Alto & proper \\
p14 & Soprano & proper \\
\hline
\end{tabular}

We recorded six female students from Music Academy in Cracow. Prior to beginning the recording session, we asked students to fill in a form, regarding their voice type and years of vocal education. We asked each singer to sing / a / vowel - rising glissando with reduced vibrato. We instructed students to use at least two registers during the exercise. Each singer repeated the glissando task until she was able to achieve register transition at least from the chest to the head. A single recording session lasted for about 10 minutes. Table 3 shows information about the recorded group for this study.

We segmented passaggio regions according to table 1 . Next, we asked an musician expert to assess the singing technique according to the following criteria:

- $\quad$ presence of vocal breaks in the region of passaggio

- $\quad$ presence of timbre differences under and above passaggio thresholds

- $\quad$ sound attack incorrectness

Based on expert's opinion we divided recordings into high (none of criteria present), or low (at least one of criteria present) quality. Figure 3 shows an egg signals with spectrograms for smooth tranistion and vocal breaks observed.

\subsection{Data acquisition}

We recorded each singer in an anechoic chamber at the University of Science and Technology in Cracow. We used a G.R.A.S. 40 AF high precision condenser microphone, which was connected to a G.R.A.S. 12AA 2-channel power module and an M-Audio PROFIRE 610 audio interface with an Octanes preamplifier to record the acoustic signal. Additionally, the electroglottographic signals were recorded using a KayPentax EGG recorder, model 6103 , connected to the second channel of the power module. We used the Adobe Audition package for recording the signal with a $44100 \mathrm{~Hz}$ sampling frequency at 16 bits. The singers were asked to keep the same distance from the microphone and standing 
posture during all recording sessions. We used the acoustic signal during expert's assessment, and in order to determine passaggio regions. We used the egg signal to observe a causal relationship between the changes in the vocal folds vibrations way and the audible acoustical image changes.

\subsection{Signal parametrization}

The main aim of this study was looking for parameters to describe physiological changes in vocal folds vibrations way during register transition. These changes cause a vocal break in the region of passaggio. This was noticed by egg amplitude reduction and sudden changes of its spectrum. This observation proved the occurance of vocal break during incorrect phonation (see 3).

We used frame-based signal analysis with a $25 \mathrm{~ms}$ frame length and 50\% overlapping. We measure fundamental frequency (F0) from the egg signal. We used the auto correlation method [15] to estimate F0. We used the COVAREP repository [16] to get all needed parametrization methods implementations. All recordings were normalized by the Root Mean Square (RMS) method to get signal power equalization. We used the Matlab environment during the signal parametrization process.

We used the Kruskal-Wallis test to test the null hypothesis, that PPC values obtained for low and high quality passaggio comes from the same distribution. This test was used because of the non-normality of the distribution.

\section{Results}
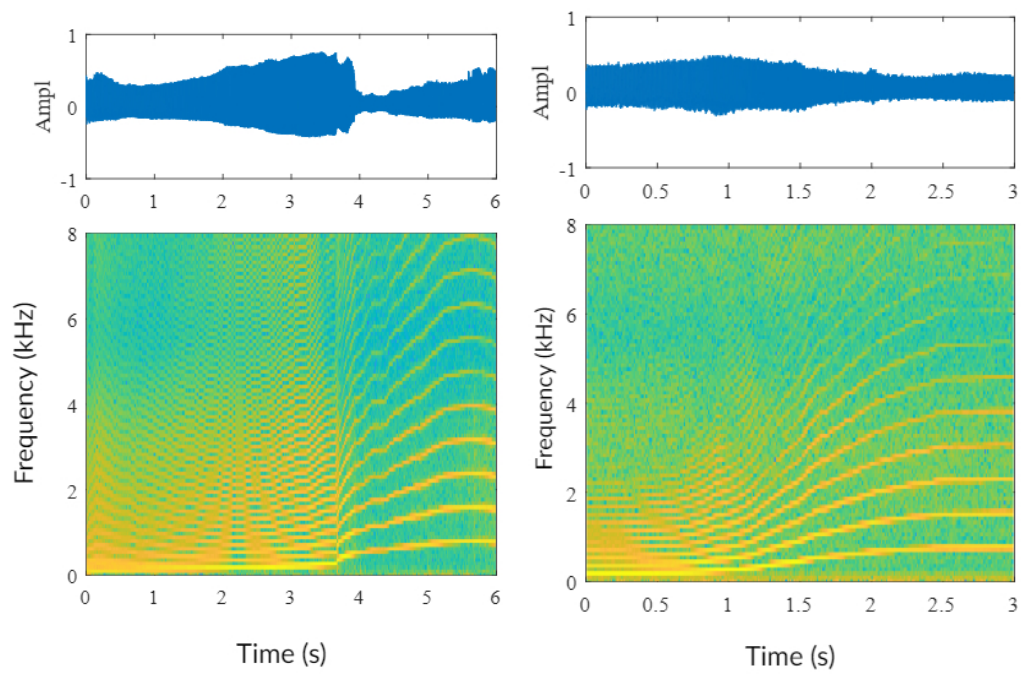

Figure 3. Register transition examples (egg and spectrocrams) for inproper (left) and proper (right) glissando.

Authors compared spectrograms and glottograms for each singer. As a result we noticed, that audible vocal breaks cause signal spectrum sudden changes in the region of passaggio. Additionally, there was noticeable amplitude change in egg present. We did not observe this sudden changes for high quality passaggio. Figure ?? presents example for vocal break and smooth transition observed in this study.

We observed sudden, instantenous peaks in pitch derivative ( $\mathrm{dF} 0 / \mathrm{dt})$ characteristics in the region of passaggio for most subjects. This observation was presented on figure 4. Additionally, we noticed that peaks in $\mathrm{dF} 0 / \mathrm{dt}$ are lower for high quality passaggio. It means, that singers with the highest expert assessment have an ability to make vocal folds vibratory pattern changes inaudible. It proves that the correct emission skills have been acquired among this group. Figure 5 shows an averaged dF0/dt for singers with low and 
high quality passaggio assesment.

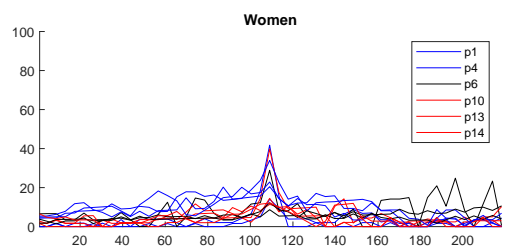

Figure 4. F0 changes for each singer.

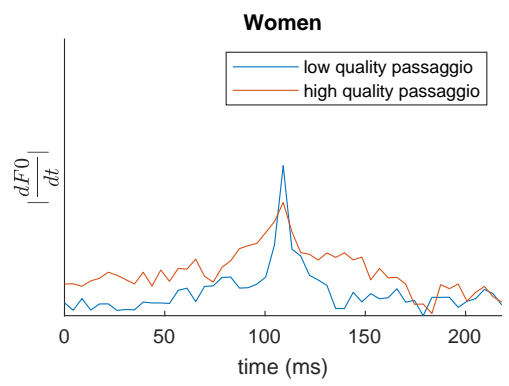

Figure 5. Average F0 changes for low and high quality passagio.

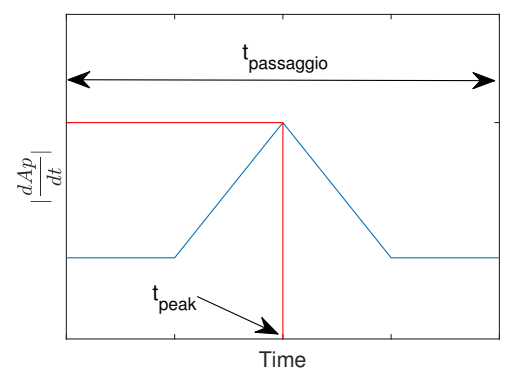

Figure 6. The concept of passaggio peak coefficient (PPC) determining for F0 vector

According to sudden, instantaneous jumps in pitch derivative characteristic during glissando we defined PPC. It describes the passaggio by location of these instantaneous jumps time moment $t_{\text {peak }}$. For this purpose, we created an specific algorithm to get values of PPC parameter for F0, that is described in detail below:

At first, the algorithm calculates the derivatives of vectors containing F0 values in subsequent time frames. Next, it is looking for the maximum peak of that derivative. This corresponds to the largest change from frame to frame of analyzing parameter derivative vector (dF0). Finally, it calculates the ratio between the height of this peak and the median derivative of the analyzed vector. The calculated factor corresponds to the Passaggio Peak Coefficient (PPC) value for the analyzed vector. Whole procedure is presented on figure 6.

$$
P P C_{F 0}=\frac{\left|d F 0\left(t_{\text {peak }}\right)\right| * N}{\sum_{n=0}^{N}|d F 0(n)|}
$$

Finally, we determined Passaggio Peak Coefficient for F0 $\left(P P C_{F 0}\right)$ for each detected transition. We analyzed the data for the classification of experience and vocal skills. According to expert classification, results were divided into two groups: low and high quality. We observed a statistically significant difference between $P P C_{F 0}(p=0,01)$ from the KruskalWallis test. Figure 7 presents $P P C_{F 0}$ median comparison for this two groups. Singers with low quality passaggio tend to achieve higher pitch jumps. 


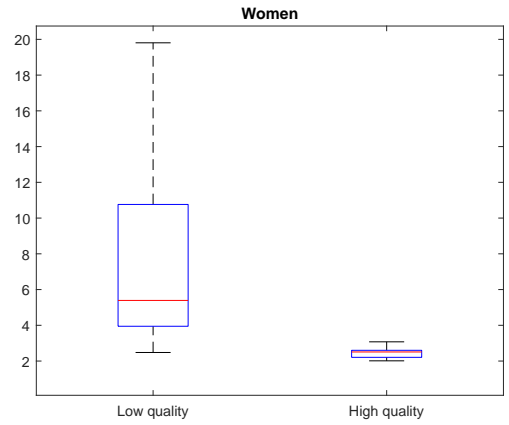

Figure 7. $\mathrm{PPC}_{F 0}$ mediancomparison forlowandhighqualitypassaggio.

\section{Discussion}

Instantaneous peak jumps are present for all studied singers. It confirms the results from $[6,10,13]$. Female singers tend to decrease the amplitudes of these jumps with vocal skills. We introduced a new parameter, called Passaggio Peak Coefficient (PPC) to show how strongly these peaks distinguish from the mean of feature vector derivative.

$P P C_{F 0}$ decreased with increasing vocal skills. That observation confirmed the hypothesis from [10] concerning the decrease of pitch jumps during vocal training. Additionally training contributes to smoothing transitions [6]. The Kruskal-Wallis test results proved differences between $P P C_{F 0}$ for low and high quality passaggio at a $5 \%$ significance level. The null hypothesis, that results come from the same distribution was rejected. This indicates the potential of the developed parameter in the process of monitoring the quality of the voice of a singer. It should be examined on a wider group of subjects in the future.

The presented results are not in line with [5], where authors did not observe any pitch jumps during passaggio. These discrepancies result from material recordings way: in the cited work, the authors used recordings of professional tenors performing modal voice to the head (falsetto) as a simulation of unprofessional voices. Recordings of the same people performing the transition from the chest record to SVAP (Stage Voice Above the Passaggio) were used to simulate skilled singers.

We used PPC to parametrize the correctness of vocal registers transition. This change is related with laryngeal mechanism transition from M1 to M2, caused by reduction in the vocal folds contact area. It causes reduction of egg amplitude, observed on ??. It is manifested by a voice register transition, and causes vocal breaks during incorrect passaggio. Additionally, we observed a noticeable reduction in egg vocal intensity after registers change. The authors will investigate this observation in future studies.

We noticed, that instantenous pitch jumps appear only for transitional notes. Unskilled singer (low quality passaggio) skip this sounds and sing the upper ones. It causes audible changes, and lack of ability to correct sing passaggio. Also, it causes changes in the higher frequencies on spectrograms.

\section{Conclusion}

This study complements previous research $[6,9,10,12,14,17]$, which examined pitch jumps during register transition. However, none of these compared the amplitude of the jumps among passaggio assesed by an expert. It is presented in the results of this article. We clearly demonstrated that with professional voice training singers can smooth register transitions through the appropriate configuration of the vocal apparatus. Additionally, it is possible to describe this transition using a newly defined parameter, PPC.

$P P C_{F 0}$ values differ between low and high quality passaggio at a $5 \%$ significance level. In the future, the authors plan to supplement the research group with skilled and mediumskilled male voices.

In this study we confirmed the hypothesis, that there is a possibility to assess voice quality by F0 derivative analyzing during passaggio. However, parameters to assess passaggio should be defined for each singer. Otherwise, the lack of a F0 peaks may indicate that the 
registry has not been changed.

In terms of further research, the issue at hand could be developed with the division of the research group into types of voice, as other studies have focused on individual types ([10] soprano, [5] - tenor) or have conducted an analysis of each registered type [6]. Also, the authors plan to perform similar PPC analyses for other parameters describing the workings of the vocal tract $(\mathrm{H} 1 \mathrm{H} 2, \mathrm{HNR}, \mathrm{NAQ}, \mathrm{PSP}$, formants etc.) and compare the results with acoustic signal analysis.

Informed Consent Statement: Informed consent was obtained from all subjects involved in the study.

\section{References}

1. du texte García, M...A. Mémoire sur la voix humaine : présenté à l'Académie des sciences en 1840 / par Manuel Garcia,... 1847.

2. Ware, C. Basics of Vocal Pedagogy: The Foundations and Process of Singing. 1998.

3. Hollien, H. On Vocal Registers; Vol. 2, 1972; p. 35.

4. Roubeau, B.; Henrich, N.; Castellengo, M. Laryngeal Vibratory Mechanisms: The Notion of Vocal Register Revisited. Journal of Voice 2009, 23, 425-438. doi:10.1016/J.JVOICE.2007.10.014.

5. Echternach, M.; Burk, F.; Köberlein, M.; Herbst, C.T.; Döllinger, M.; Burdumy, M.; Richter, B. Oscillatory Characteristics of the Vocal Folds Across the Tenor Passaggio. Journal of Voice 2017, 31. doi:10.1016/j.jvoice.2016.06.015.

6. Henrich, N.; D'Alessandro, C.; Doval, B.; Castellengo, M. Glottal open quotient in singing: measurements and correlation with laryngeal mechanisms, vocal intensity, and fundamental frequency. The Journal of the Acoustical Society of America 2005, 117, 1417-30.

7. Sundberg, J.; Högset, C. Voice source differences between falsetto and modal registers in counter tenors, tenors and baritones. Logopedics, phoniatrics, vocology 2001, 26, 26-36.

8. Echternach, M.; Traser, L.; Richter, B. Vocal Tract Configurations in Tenors' Passaggio in Different Vowel Conditions-A Real-Time Magnetic Resonance Imaging Study. Journal of Voice 2014, 28. doi:10.1016/j.jvoice.2013.10.009.

9. Svec, J.; Schutte, H.; Miller, D. On pitch jumps between chest and falsetto registers in voice: Data from living and excised human larynges. The Journal of the Acoustical Society of America 1999, 106, 1523-1531. doi:10.1121/1.427149.

10. Echternach, M.; Burk, F.; Köberlein, M.; Selamtzis, A.; Döllinger, M.; Burdumy, M.; Richter, B.; Herbst, C.T. Laryngeal evidence for the first and second passaggio in professionally trained sopranos. PLOS ONE 2017, 12, e0175865. doi:10.1371/journal.pone.0175865.

11. Miller, D.G.; Svec, J.G.; Schutte, H.K. Measurement of characteristic leap interval between chest and falsetto registers. Journal of voice : official journal of the Voice Foundation 2002, 16, 8-19.

12. Tokuda, I.T.; Zemke, M.; Kob, M.; Herzel, H. Biomechanical modeling of register transitions and the role of vocal tract resonators. The Journal of the Acoustical Society of America 2010, 127, 1528-1536. doi:10.1121/1.3299201.

13. Bernardoni, N.H.; Roubeau, B.; Castellengo, M. On the use of electroglottography for characterisation of the laryngeal mechanisms 2003. p. 1.

14. Roubeau, B.; Chevrie-Muller, C.; Arabia-Guidet, C. Electroglottographic Study of the Changes of Voice Registers. Folia Phoniatrica et Logopaedica 1987, 39, 280-289. doi:10.1159/000265871.

15. Kumar, S.; Bhattacharya, S.; Patel, P. A new pitch detection scheme based on ACF and AMDF. 2014 IEEE International Conference on Advanced Communications, Control and Computing Technologies 2014, pp. 1235-40.

16. Degottex, G.; Kane, J.; Drugman, T.; Raitio, T.; Scherer, S. COVAREP - A collaborative voice analysis repository for speech technologies. 2014 IEEE International Conference on Acoustics, Speech and Signal Processing (ICASSP), 2014, pp. 960-964.

17. Zañartu, M.; Mehta, D.D.; Ho, J.C.; Wodicka, G.R.; Hillman, R.E. Observation and analysis of in vivo vocal fold tissue instabilities produced by nonlinear source-filter coupling: a case study. The Journal of the Acoustical Society of America 2011, 129, 326-39. doi:10.1121/1.3514536. 
